\title{
Evaluated Excited-State Time-Independent Correlation Function and Eigenfunction of the Harmonics Oscillator Cosine Asymmetric Potential via Numerical Shooting Method
}

\author{
Artit Hutem $^{1,2}$ and Piyarut Moonsri ${ }^{3}$ \\ ${ }^{1}$ Physics Division, Faculty of Science and Technology, Phetchabun Rajabhat University, Phetchabun 67000, Thailand \\ ${ }^{2}$ Department of Physics, Faculty of Science, Kasetsart University, Bangkok 10900, Thailand \\ ${ }^{3}$ Chemistry Division, Faculty of Science and Technology, Phetchabun Rajabhat University, Phetchabun 67000, Thailand \\ Correspondence should be addressed to Artit Hutem; magoohootem@yahoo.com
}

Received 6 August 2014; Revised 5 January 2015; Accepted 12 January 2015

Academic Editor: Angel Rubio

Copyright ( 2015 A. Hutem and P. Moonsri. This is an open access article distributed under the Creative Commons Attribution License, which permits unrestricted use, distribution, and reproduction in any medium, provided the original work is properly cited.

\begin{abstract}
We aimed to evaluate the ground-state and excite-state energy eigenvalue (En), wave function, and the time-independent correlation function of the atomic density fluctuation of a particle under the harmonics oscillator Cosine asymmetric potential (Saad et al. 2013). Instead of using the 6-point kernel of 4 Green's function (Cherroret and Skipetrov, 2008), averaged over disorder, we use the numerical shooting method (NSM) to solve the Schrödinger equation of quantum mechanics system with Cosine asymmetric potential. Since our approach does not use complicated formulas, it requires much less computational effort when compared to the Green functions techniques (Cherroret and Skipetrov, 2008). We show that the idea of the program of evaluating time-independent correlation function of atomic density is underdamped motion for the Cosine asymmetric potential from the numerical shooting method of this problem. Comparison of the time-independent correlation function obtained from numerical shooting method by Boonchui and Hutem (2012) and correlation function experiment by Kasprzak et al. (2008). We show the intensity of atomic density fluctuation $(\delta n(x)=\widetilde{n}(x)-\widetilde{m}(x))$ in harmonics oscillator Cosine asymmetric potential by numerical shooting method.
\end{abstract}

\section{Introduction}

Most problems encountered in quantum mechanics cannot be solved exactly. Exact solutions of the Schrödinger equation exist only for a few idealized systems. To solve general problems, one must resort to approximation methods. A variety of such methods have been developed, and each has its own area of applicability. There exist several means to study them, for example, Wentzel-Kramers-Brillouin [1], perturbation [2], the quasilinearization method [3], the variational method [4], function analysis $[5,6]$, the eigenvalue moment method [7], the analytical transfer matrix method [8-10], and numerical shooting method $[11,12]$.

Grobe et al. 1994 [13] proposed a criterion to determine the numerical degree of global correlation function of the multiparities quantum system. They applied this method to several situations, including electron-atom scattering and strong-field photoionization. Lye et al. 2005 [14] discussed the effect of a weak random potential, indicated by stripes in the expanded density profile of the Bose-Einstein Condensate and damped dipole oscillations. Henseler and Shapiro 2008 [15] defined the disorder-induced intensity-intensity correlation function, $C_{\omega}\left(\mathbf{r}, \mathbf{r}^{\prime}\right)=\overline{\left|\psi_{\omega}^{*}(\mathbf{r}) \psi_{\omega}\left(\mathbf{r}^{\prime}\right)\right|^{2}}$, for the BoseEinstein Condensate for Fermi gas. Cherroret and Skipetrov 2008 [16] showed decay of the average atomic density $\left(\overline{n(\mathbf{r}, t)}=\overline{|\psi(\mathbf{r}, t)|^{2}}\right)$ as a function of time. The density reaches a maximum at the arrival time $t_{\text {arrival }} \simeq 2 z^{2} / D_{\mu}$, where $D_{\mu}$ is the diffusion coefficient in random potentials. Now, a few works have concerned the expansion of Bose-Einstein Condensate in three-dimensional potentials and evaluate correlation function. Cherroret and Skipetrov 2009 [17] had shown the typical diffusion coefficient of the Bose-Einstein Condensate in a three-dimensional random potential. Beilin et al. 2010 [18] considered diffusion of cold-atomic Fermi gas in the presence of a random optical speckle potential. 
Pezze et al. 2011 [19] numerically studied the dynamics regimes of classical transport of cold atoms gases in a twodimensional anisotropic disorder potential. In this paper, we consider approximation methods that deal with stationary states corresponding to time-independent Hamiltonian. To study problem of stationary states, we focus on one approximation method: numerical shooting method useful to evaluate wave function and time-independent correlation function of a particle around attraction by the harmonics oscillator with Cosine asymmetric potential. The scheme of the paper is as follows. In Section 2, we write the basic time-independent Schrödinger equation in terms of finite difference and the harmonics oscillator Cosine asymmetric potential in terms of the new variable is given by

$$
\begin{array}{r}
\psi_{i+1}=2 \psi_{i}-\psi_{i-1} \\
-(\Delta \xi)^{2}\left(\varepsilon-\xi^{2}-2 c \xi-2 a \cos (b \xi)\right) \psi_{i} \\
i=2,3,4, \ldots
\end{array}
$$

where $2 c \xi+2 a \cos (b \xi)$ is the Cosine asymmetric potential. In Section 3, we show the idea of writing a program for evaluating energy eigenvalue wave function and correlation function of atomic density for the Cosine asymmetric potential via the numerical shooting method (Asaithambi, Ledoux and van Daele, Boonchui and Hutem $[12,20,21])$. Section 4 contains our conclusions.

\section{Time-Independent Schrödinger Equation in Finite Difference Formula for Harmonics Oscillator Cosine Asymmetric Potential}

We consider a particle of mass $\mu$ moving on the $x$-axis in a time-independent potential $\mathscr{V}(x)$. The time-independent Schrödinger equation corresponding to this one-dimensional motion is

$$
-\frac{\hbar^{2}}{2 \mu} \frac{d^{2} \psi_{n}(x)}{d x^{2}}+\mathscr{V}(x) \psi_{n}(x)=\mathscr{E}_{n} \psi_{n}(x)
$$

where $\mathscr{C}_{n}$ is the total energy eigenvalues of the particle. The solution of this equation yields the allowed energy eigenvalues $\mathscr{E}_{n}$ and the corresponding wave function $\psi_{n}(x)$. To solve this partial differential equation, we need to specify the potential $\mathscr{V}(x)$ as well as the boundary condition; the boundary condition can be obtained from the physical requirement of the system.

Suppose a particle is bound state to around of attraction by the harmonics oscillator Cosine asymmetric potential (see Figure 1):

$$
\mathscr{V}(x)=\frac{1}{2} \mu \omega^{2} x^{2}+c x+a \cos (b x)
$$

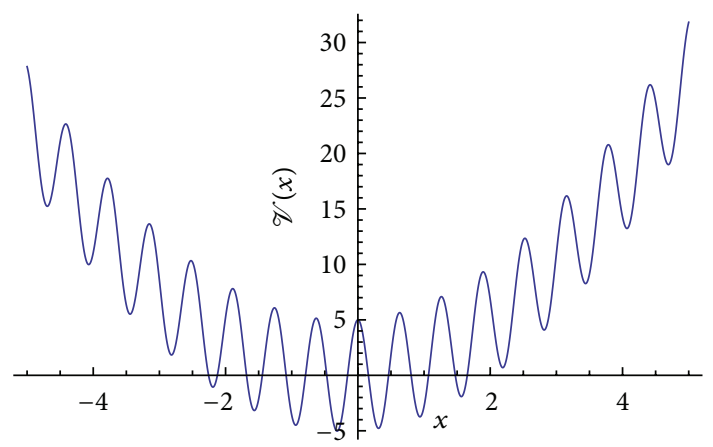

FIGURE 1: The harmonics oscillator potential is perturbed by Cosine asymmetric potential, with $a=5, b=10$, and $c=0.4$.

with $c x+a \cos (b x)$ that is called the Cosine asymmetric potential [22], where $c, a$, and $b$ are positive constants. Substituting the harmonics oscillator Cosine asymmetric potential from (3) into (2) leads to the following equation:

$$
\begin{aligned}
\mathscr{E}_{n} \psi_{n}(x)= & -\frac{\hbar^{2}}{2 \mu} \frac{d^{2} \psi_{n}(x)}{d x^{2}} \\
& +\left(\frac{1}{2} \mu \omega^{2} x^{2}+c x+a \cos (b x)\right) \psi_{n}(x) .
\end{aligned}
$$

For our approach (4) can be solved in the numerical shooting method. It is convenient to simplify the arithmetic involved in the shooting solution. We define some new dimensionless variables. Then the position variable $x$ is replaced with the dimensionless variable $\xi$ :

$$
\xi \equiv \sqrt{\frac{\mu \omega}{\hbar}} x, \quad \xi^{2}=\frac{\mu \omega}{\hbar} x^{2} .
$$

With this definition, the second-derivative term can be written as

$$
\frac{d^{2}}{d \xi^{2}}=\frac{\hbar}{\mu \omega} \frac{d^{2}}{d x^{2}}
$$

Substituting in for $x$ in terms of $\xi$ and setting $\varepsilon=2 E / \hbar \omega$ and setting $\hbar=\mu=\omega=1$ into (4), we can rewrite the timeindependent Schrödinger equation completely in terms of $\xi$ as follows:

$$
\frac{d^{2} \psi_{n}(x)}{d \xi^{2}}+\left(\varepsilon-\xi^{2}-2 c \xi-2 a \cos (b \xi)\right) \psi_{n}(x)=0 .
$$

Also, the time-independent potential in terms of the new variable is given by

$$
\mathscr{V}(\xi)=\xi^{2}+2 c \xi+2 a \cos (b \xi) .
$$

For the finite difference method, (7) is rewritten in the form of many small segment $\Delta \xi$ in the $\xi$ domain length. The secondderivative of the first term in (7) can be approximated in finite difference form as follows:

$$
\frac{d^{2} \psi_{n}(x)}{d \xi^{2}} \approx \frac{\psi_{i+1}+\psi_{i-1}-2 \psi_{i}}{(\Delta \xi)^{2}} .
$$




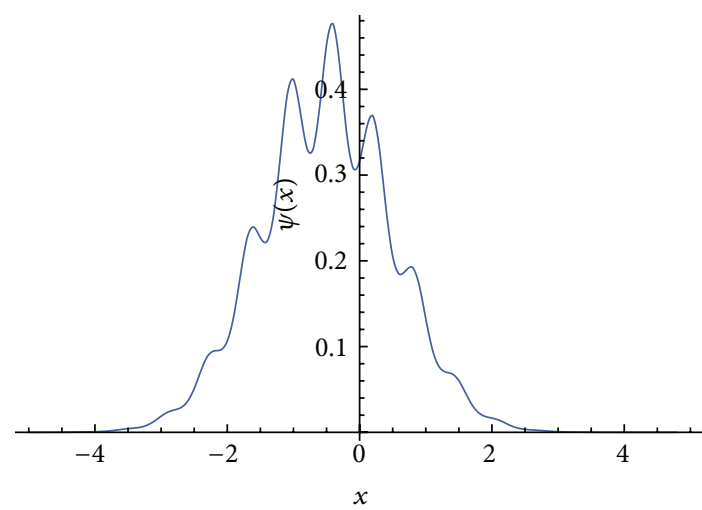

(a) En $=-0.562660496449, c=0.4, a=8, b=10.0$, and $n=0$

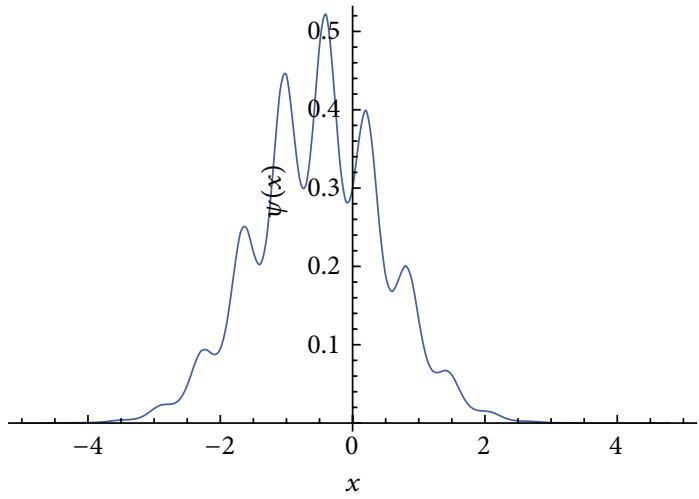

(c) En $=-2.266994942969, c=0.4, a=12, b=10.0$, and $n=0$

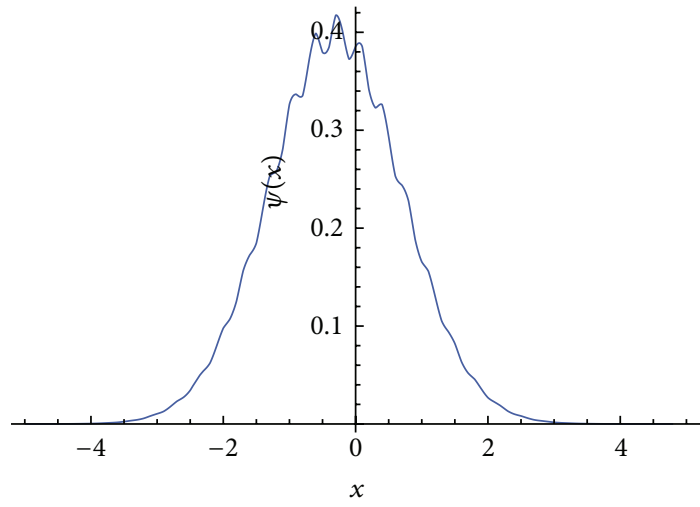

(e) En $=0.664583592675, c=0.2, a=6, b=18.0$, and $n=0$

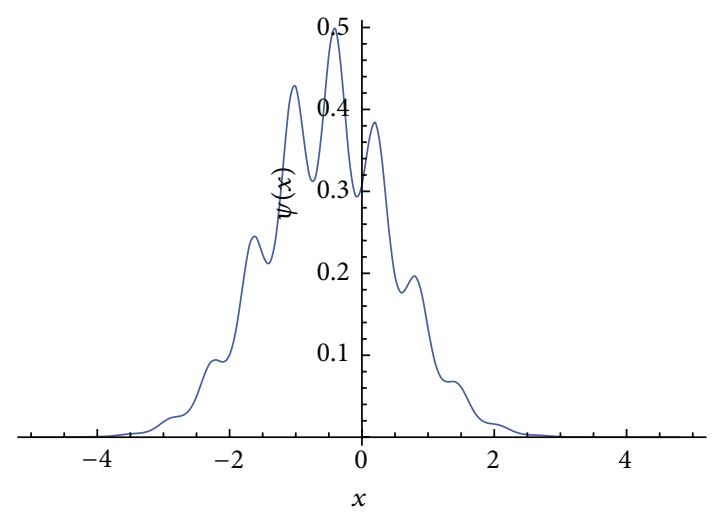

(b) En $=-1.335972183092, c=0.4, a=10, b=10.0$, and $n=0$

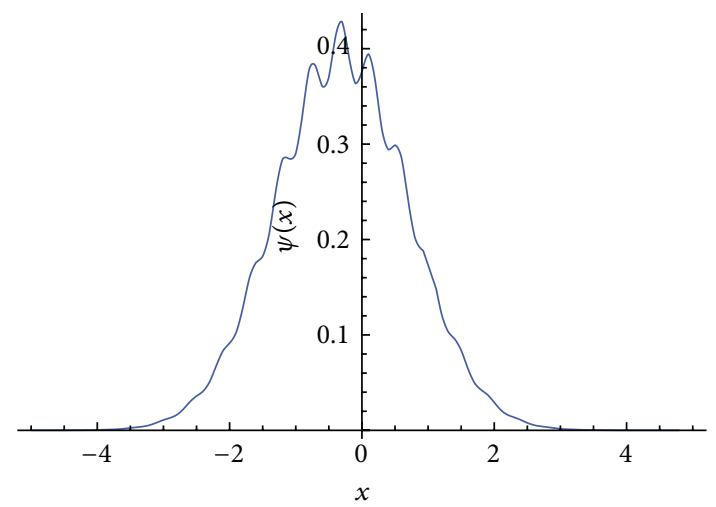

(d) En $=0.522331835003, c=0.2, a=6, b=14.0$, and $n=0$

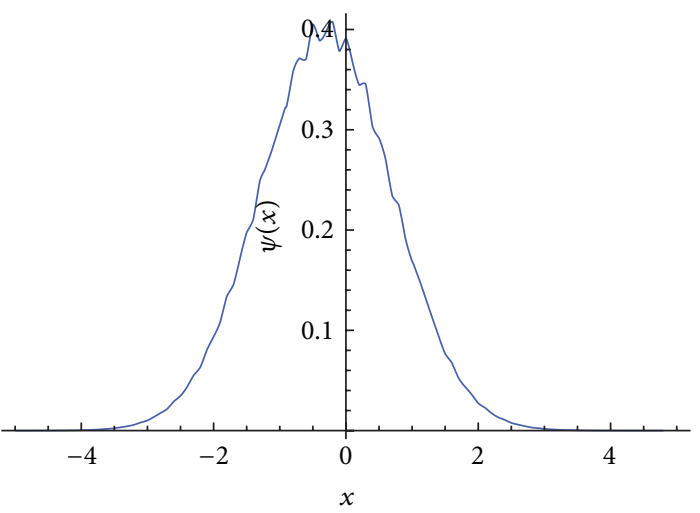

(f) En $=0.751492211595, c=0.2, a=6, b=24.0$, and $n=0$

FIGURE 2: Figures (a)-(c) show plot of the time-independent wave function for the ground-state energy $(n=0)$ in case of varying $a=8,10$, 12 (the amplitude of barrier potential) in harmonics oscillator Cosine asymmetric potential. Figures (d)-(f) show the wave function for the ground-state energy with varying $b=14,18,24$ harmonics oscillator Cosine asymmetric potential.

We can obtain the form of the time-independent Schrödinger equation in terms of finite difference by substituting (9) into (7), and we get

$$
\begin{array}{r}
\psi_{i+1}=2 \psi_{i}-\psi_{i-1} \\
-(\Delta \xi)^{2}\left(\varepsilon-\xi^{2}-2 c \xi-2 a \cos (b \xi)\right) \psi_{i} \\
i=2,3,4, \ldots
\end{array}
$$

where $\xi_{i+1}=\Delta \xi+\xi_{i}$. The special potential given by harmonics oscillator Cosine asymmetric potential has been used in calculating (10) in the mathematica program (see Section 3).

\section{Numerical Shooting Method and Results}

We construe the new variable to be used in calculating the ground-state energy eigenvalue, wave function, and the timeindependent correlation function of the harmonics oscillator Cosine asymmetric potential. 


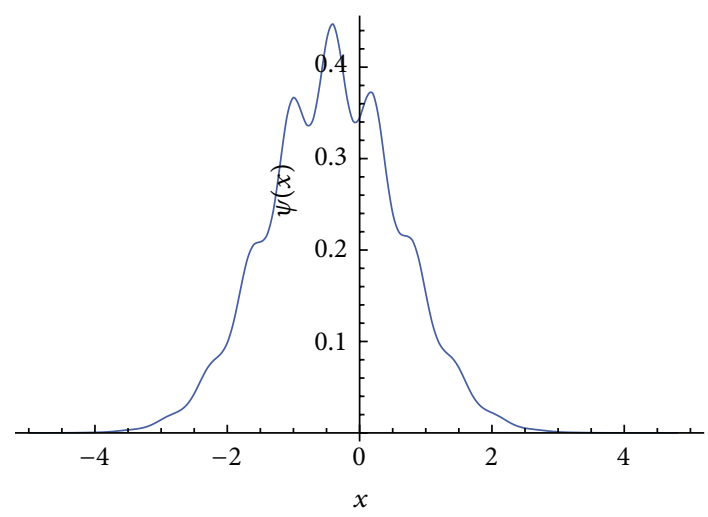

(a) En $=0.357420646119, c=0.3, a=5, b=10.0$, and $n=0$

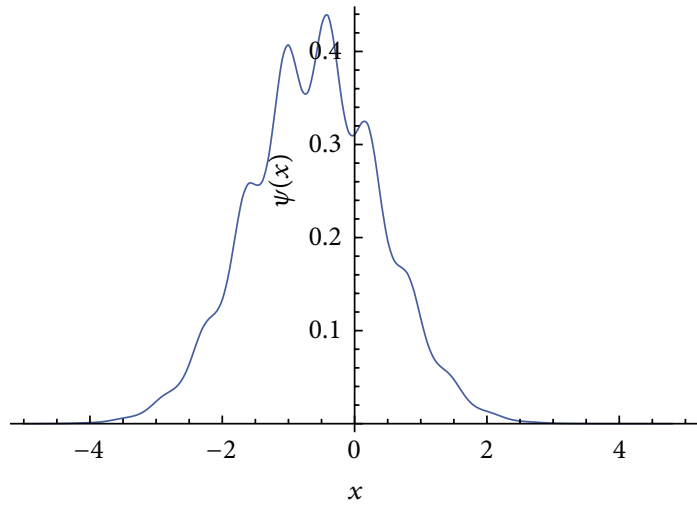

(c) En $=0.197420666459, c=0.5, a=5, b=10.0$, and $n=0$

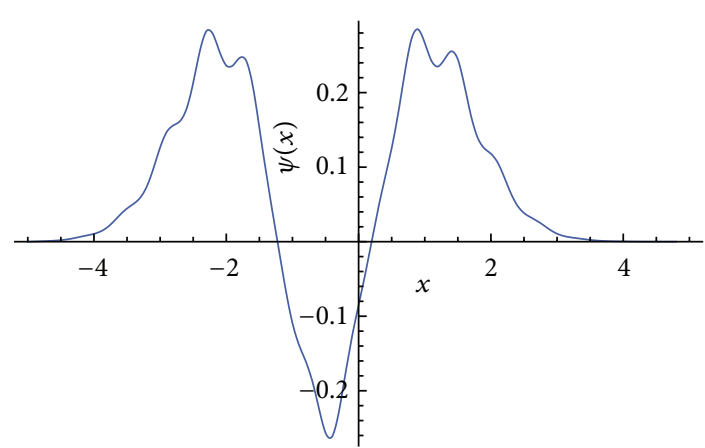

(e) En $=4.229409700958, c=0.4, a=5, b=10.0$, and $n=2$

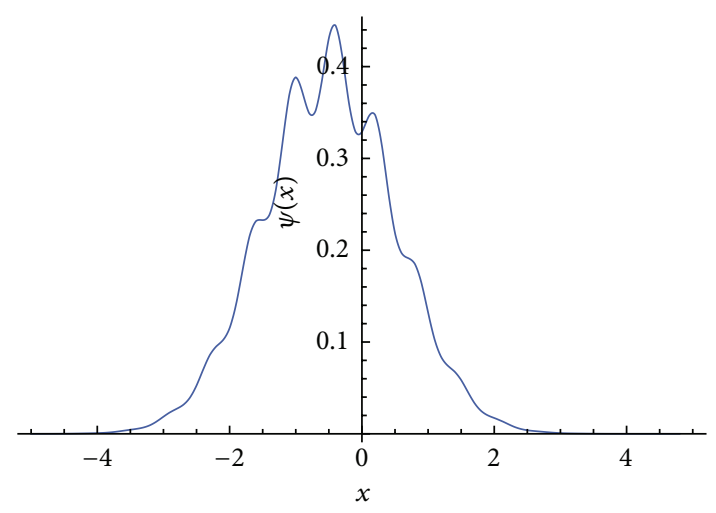

(b) En $=0.287420653214, c=0.4, a=5, b=10.0$, and $n=0$

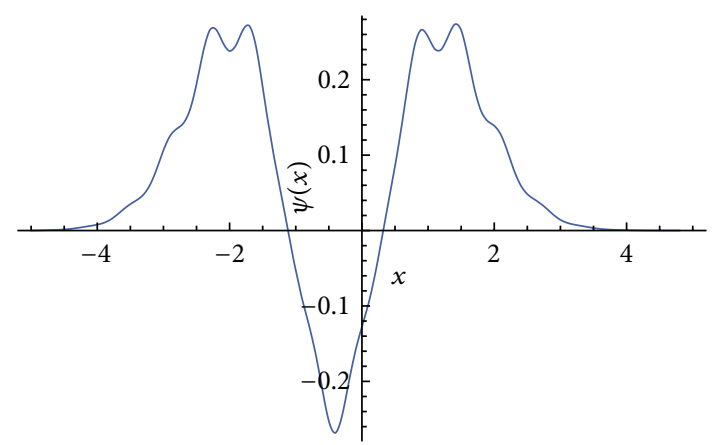

(d) En $=4.299406788515, c=0.3, a=5, b=10.0$, and $n=2$

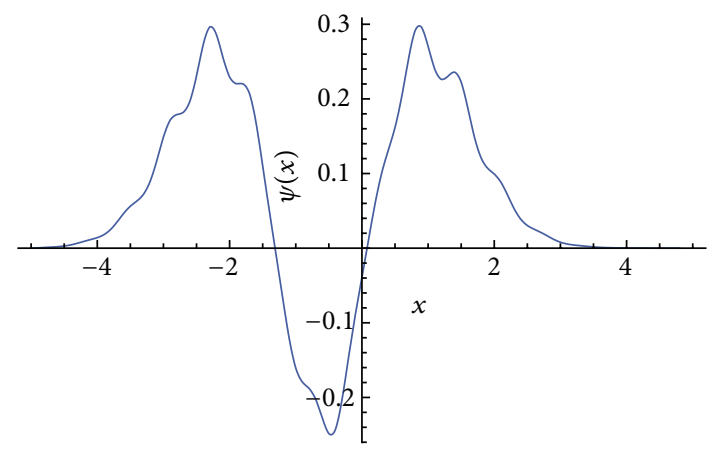

(f) En $=4.139415930374, c=0.5, a=5, b=10.0$, and $n=2$

FIGURE 3: Schematic representation for behavior of the wave function for the ground-state energy $(n=0)$ and the second excited-state energy $(n=2)$ in case of the harmonics oscillator Cosine asymmetric potential with varying $c=0.3,0.4,0.5$.

(1) $\xi_{\min }$ is the start position in the analysis range.

(2) $\xi_{\max }$ is the ultimate position in the analysis range.

(3) $\xi$ is any position in the analysis range.

(4) $n n$ is a number of very small bars in the analysis range.

(5) $\Delta \xi$ is the length of very small bars so that

$$
\Delta \xi=\frac{\xi_{\max }-\xi_{\min }}{n n} .
$$

The logic of the numerical shooting method evaluation of energy eigenvalue, eigenfunction, and time-independent correlation function for the harmonics oscillator Cosine asymmetric potential is as follows.

(i) Input values $\xi_{\min }$ and $\xi_{\max }$ in mathematica program for the harmonics oscillator Cosine asymmetric potential.

(ii) Input the period amount.

(iii) Input (10) into mathematica program. 


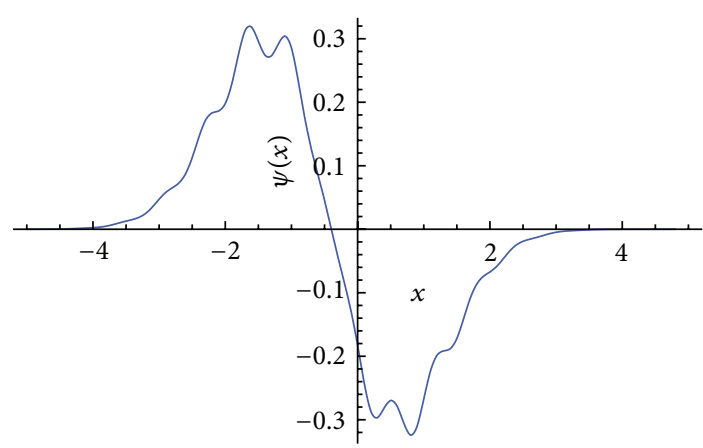

(a) En $=2.331415049731, c=0.3, a=5, b=10$, and $n=1$

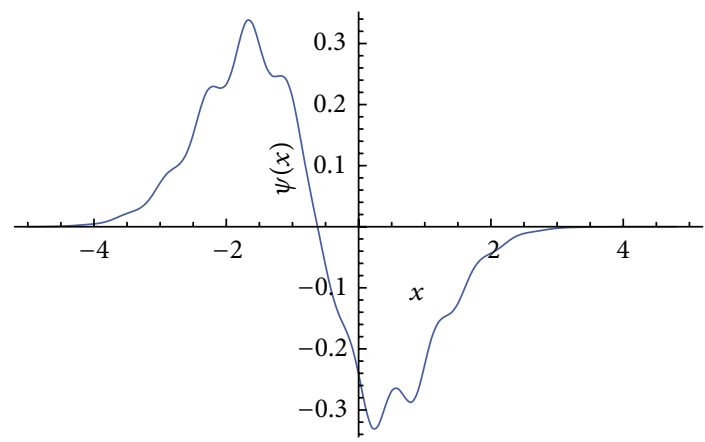

(c) En $=2.171415559947, c=0.5, a=5, b=10$, and $n=1$

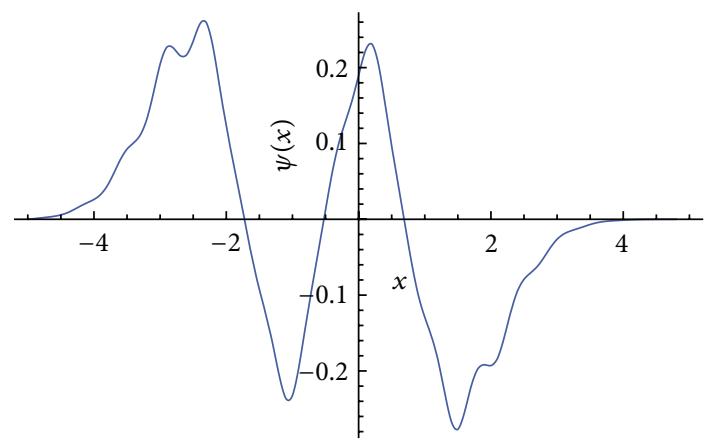

(e) En $=6.190472530201, c=0.4, a=5, b=10$, and $n=3$

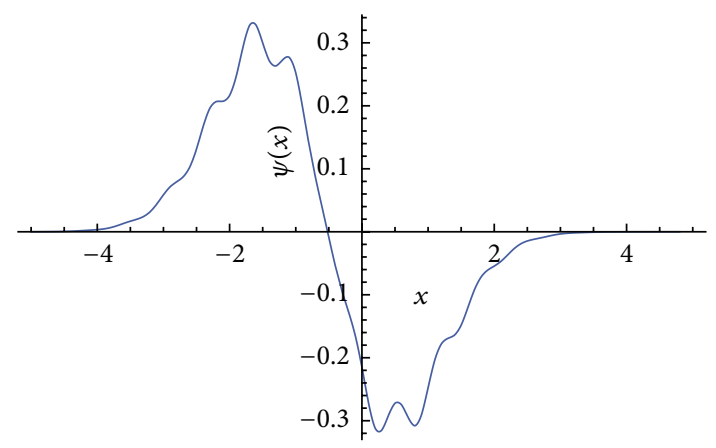

(b) En $=2.261415204498, c=0.4, a=5, b=10$, and $n=1$

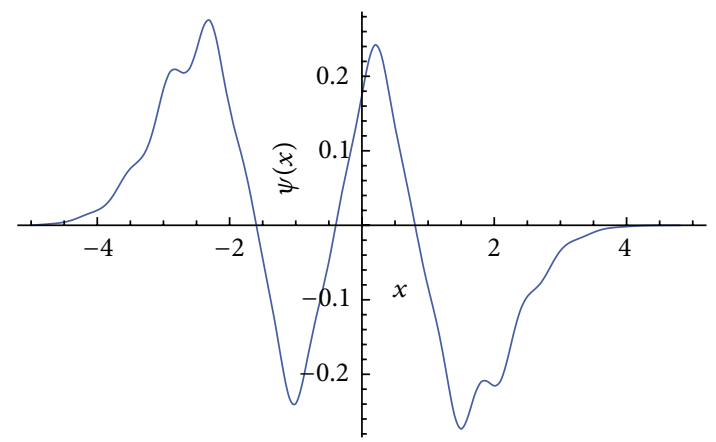

(d) En $=6.260444417595, c=0.3, a=5, b=10$, and $n=3$

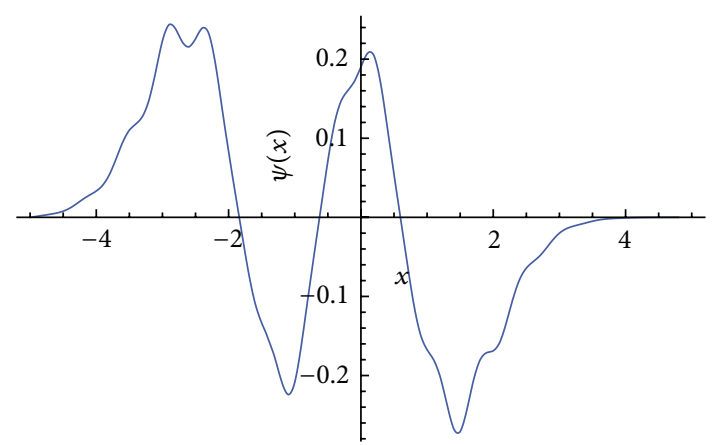

(f) En $=6.100527633912, c=0.5, a=5, b=10$, and $n=3$

FIGURE 4: Plot of the wave function for the first excited-state energy $(n=1)$ and the third excited-state energy $(n=3)$ in case of the harmonics oscillator Sine asymmetric potential with varying $c=0.3,0.4,0.5$.

Find the initial value for calculation. Input the initial condition by setting $\psi_{1}=0$ for the position imprisons and set $d \psi / d \xi=1$ from the slope of positions 1 and 2, so that

$$
\frac{d \psi}{d \xi} \approx \frac{\psi_{2}-\psi_{1}}{\Delta \xi} \Longrightarrow \psi_{2} \approx \Delta \xi
$$

By inputting $\psi_{1}$ and $\psi_{2}$ as two initial values for calculation, we can find $\psi_{3}$ from (10). In the same way, we can find $\psi_{4}$ by substituting $\psi_{2}$ and $\psi_{3}$ in the equation. As we keep doing this, we can find $\psi_{n}$ (see Figure 2 in [12]).

(i) The next task is to calculate wave function in (10) $\left(\psi_{i+1}\right)$ so that it approaches zero as closely as desired. Normally, we assign a small value as the standard to make sure that wave function in (10) gets close enough to zero. For example, if $\left|\psi_{i+1}\right| \leq 10^{-6}$, we stop the calculation and accept the final energy as the numerical solution.

(ii) Plot the wave function by the graph related to $i$.

(iii) Plot the wave function that is normalized by the graph related to $i$.

(iv) Plot the probability of the average atomic density $\widetilde{n}(x)=\overline{|\psi(x)|^{2}}$ for the harmonics oscillator Cosine asymmetric potential.

(v) Input values $\xi_{\min }$ and $\xi_{\max }$ in the mathematica program for the harmonics oscillator potential.

(vi) Input equation $\psi_{i+1}=2 \psi_{i}-\psi_{i-1}-(\Delta \xi)^{2}\left(\varepsilon-\xi^{2}\right) \psi_{i}$ into the mathematica program for the harmonics oscillator potential.

(vii) For example, if $|\psi(x)| \leq 10^{-6}$, we stop the evaluation and accept the final energy as the numerical solution. 


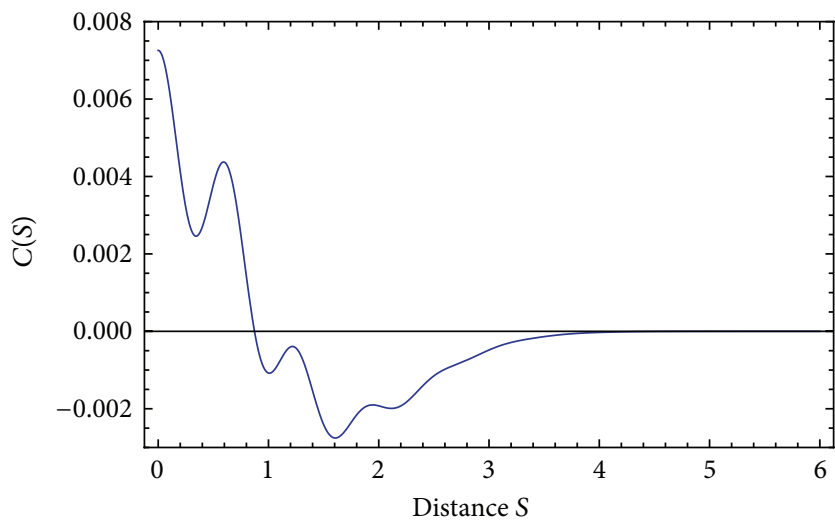

(a) $C(S)=0.007, C(S)=-0.0025, c=0.4, a=8, b=10$, and $n=0$

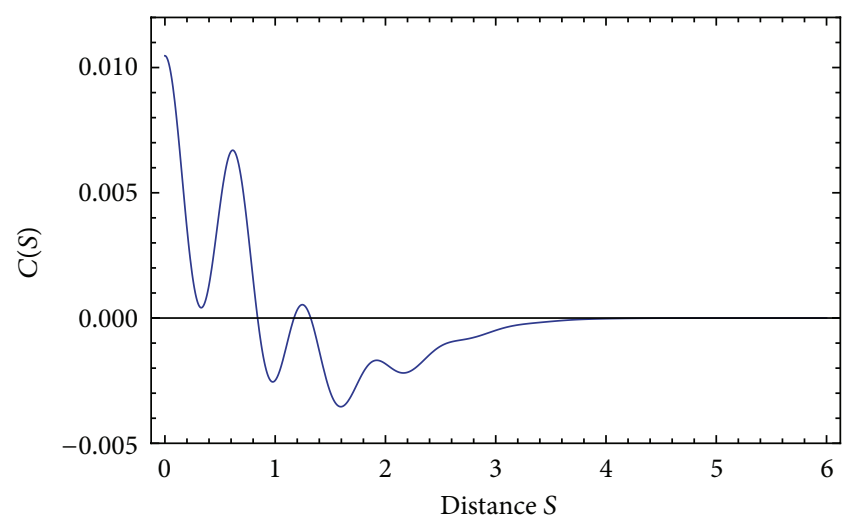

(c) $C(S)=0.010, C(S)=-0.004, c=0.4, a=12, b=10$, and $n=0$

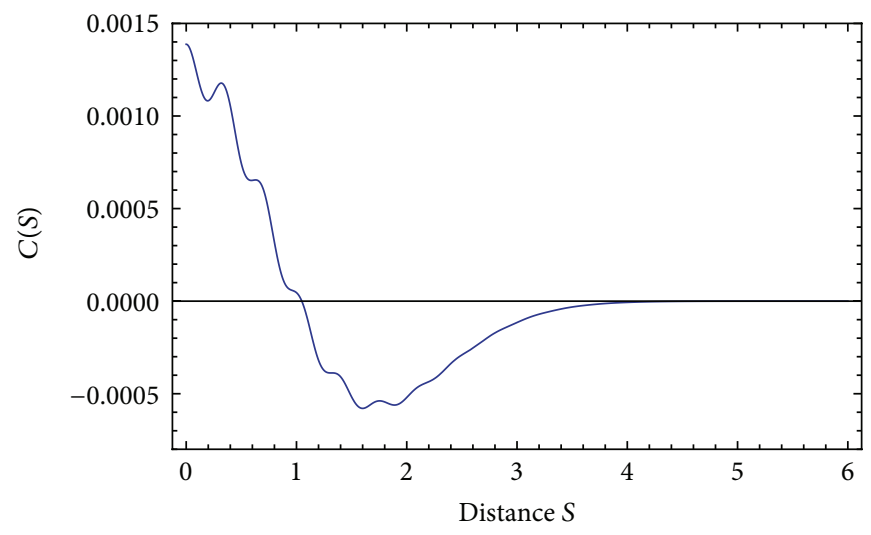

(e) $C(S)=0.0014, C(S)=-0.0006, c=0.2, a=6, b=18$, and $n=0$

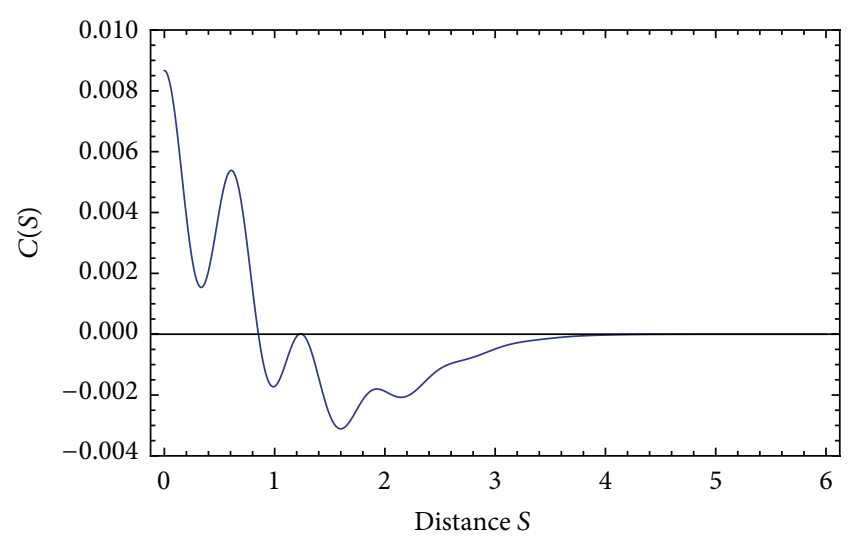

(b) $C(S)=0.0085, C(S)=-0.003, c=0.4, a=10, b=10$, and $n=0$

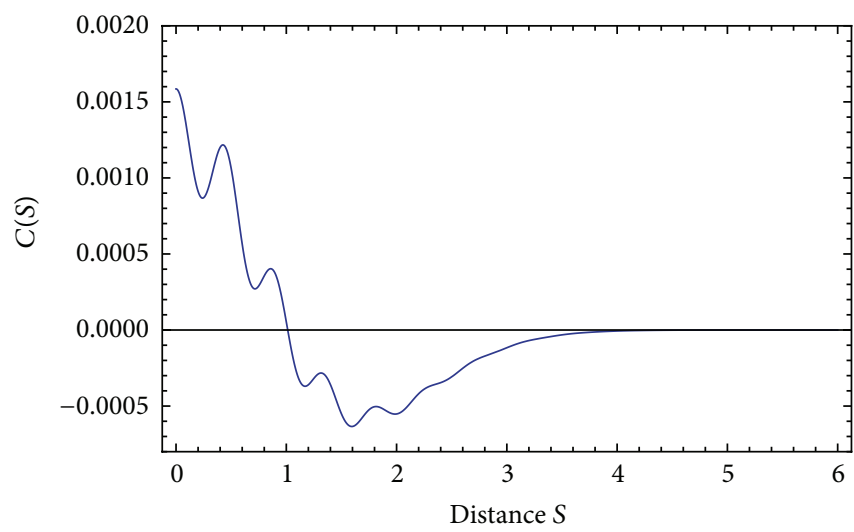

(d) $C(S)=0.0016, C(S)=-0.0007, c=0.2, a=6, b=14$, and $n=0$

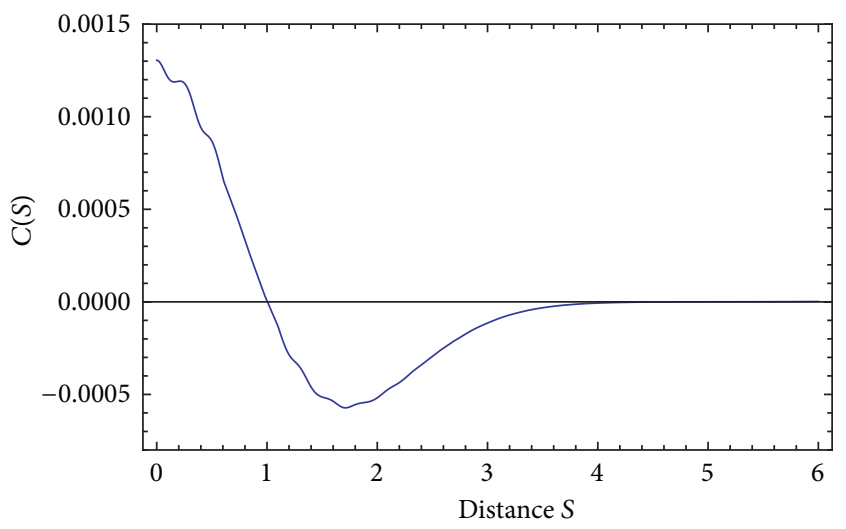

(f) $C(S)=0.0013, C(S)=-0.0006, c=0.2, a=6, b=24$, and $n=0$

FIGURE 5: Figures (a)-(c) show plot of the time-independent correlation function for the ground-state energy $(n=0)$ in case of varying $a=8$, 10,12 (the amplitude of barrier potential) in harmonics oscillator Cosine asymmetric potential. Figures (d)-(f) show the correlation function for the ground-state energy with varying $b=14,18,24$ harmonics oscillator Cosine asymmetric potential.

(viii) Plot the wave function that is normalized for the harmonics oscillator potential by the graph related to $i$.

(ix) Plot the probability of the average atomic density $\widetilde{m}(x)=\overline{|\psi(x)|^{2}}$ for the harmonics oscillator potential.

(x) Plot the time-independent atomic density fluctuation $\delta n(x)=\widetilde{n}(x)-\widetilde{m}(x)[16]$ by the graph related to $i$. (xi) Plot the time-independent correlation function $C(x, \dot{x})=\overline{\delta n(x) \delta n(\dot{x})} / \overline{n(x) n(\dot{x})}[16]$.

\section{Conclusion}

In conclusion, we then represented the method by obtaining numerical solution of the one-dimensional harmonic oscillator, perturbed from a set of the Cosine asymmetric potentials. 


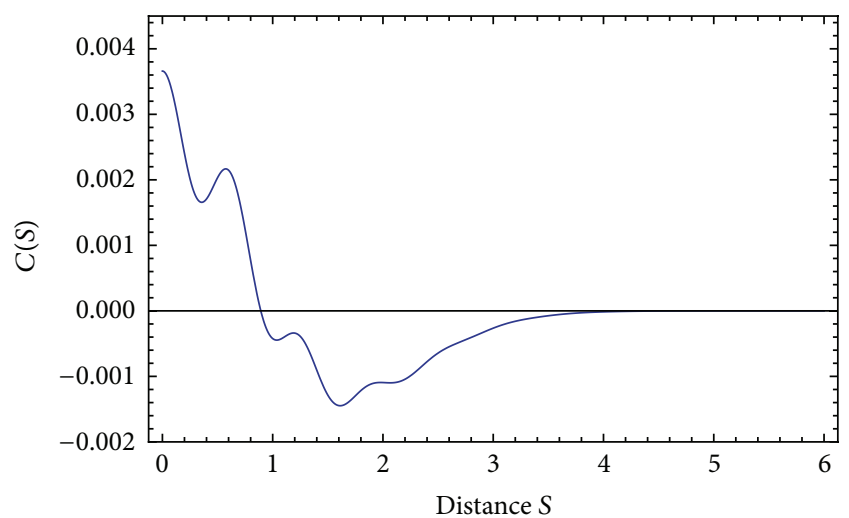

(a) $C(S)=0.0036, C(S)=-0.0014, c=0.3, a=5, b=10$, and $n=0$

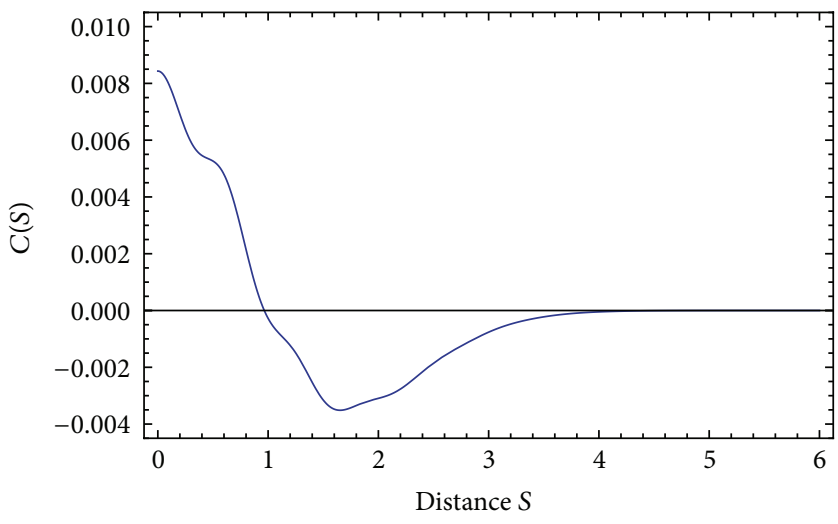

(c) $C(S)=0.0085, C(S)=-0.0055, c=0.5, a=5, b=10$, and $n=0$

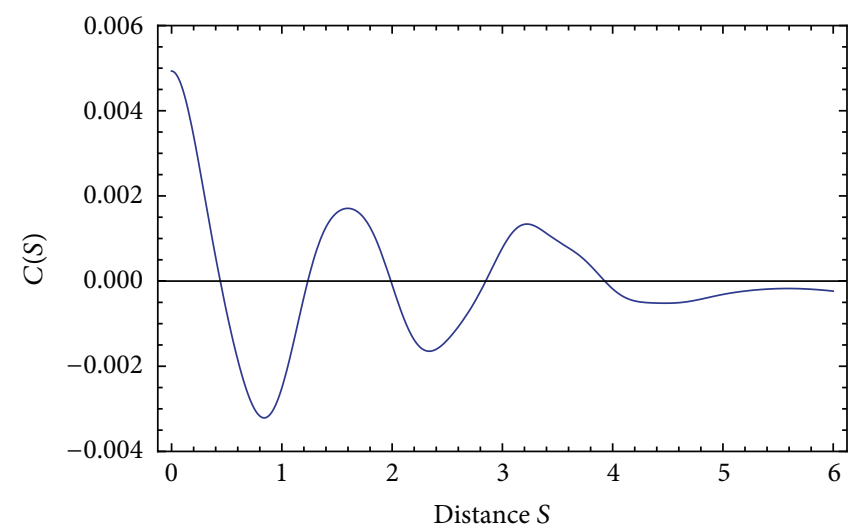

(e) $C(S)=0.005, C(S)=-0.0035, c=0.4, a=5, b=10$, and $n=2$

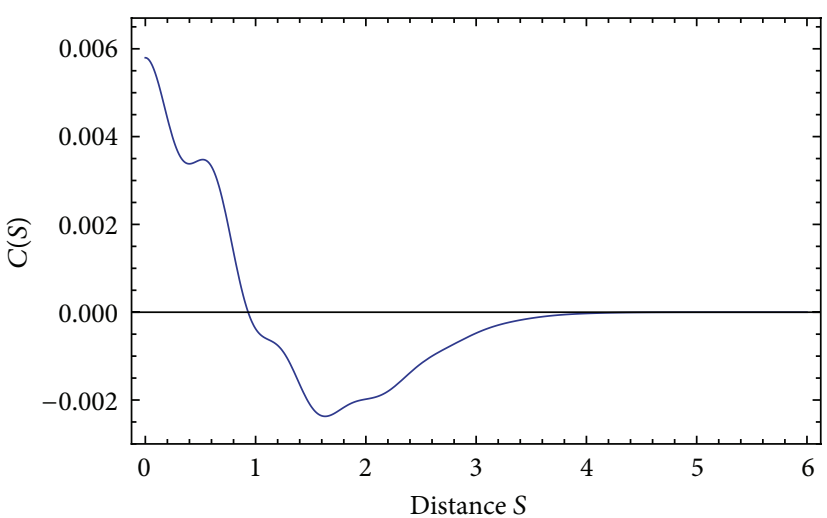

(b) $C(S)=0.006, C(S)=-0.0025, c=0.4, a=5, b=10$, and $n=0$

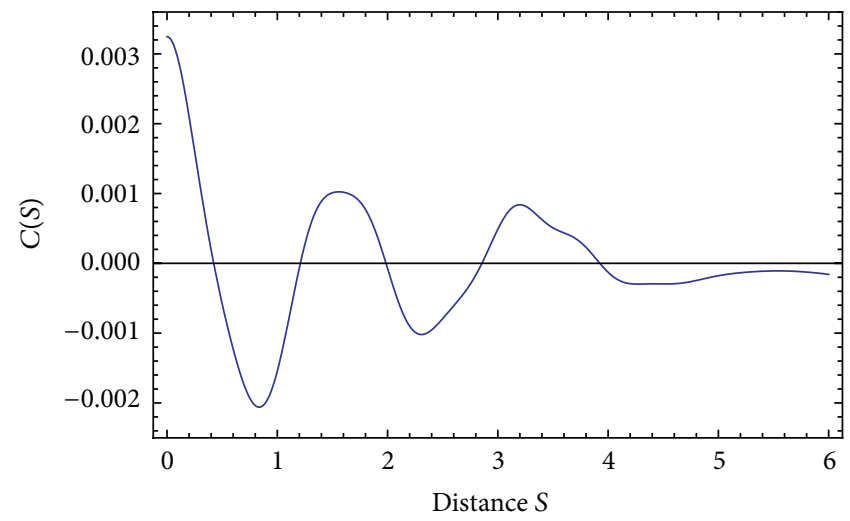

(d) $C(S)=0.0032, C(S)=-0.002, c=0.3, a=5, b=10$, and $n=2$

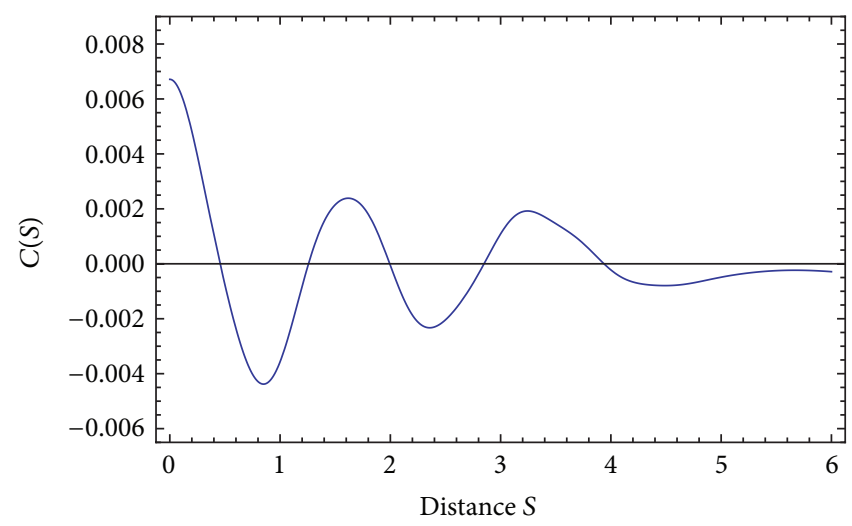

(f) $C(S)=0.0065, C(S)=-0.0045, c=0.5, a=5, b=10$, and $n=2$

FiguRE 6: Schematic representation for behavior of the time-independent correlation function for the ground-state energy $(n=0)$ and the second excited-state energy $(n=2)$ in case of the harmonics oscillator Cosine asymmetric potential with varying $c=0.3,0.4,0.5$.

In our calculation, we can obtain the time-independent correlation function corresponding with the Green functions techniques [16]. Although the numerical shooting method does not use complicated formulas, it requires much less computational effort when compared to the Green functions techniques. Generally, regarded as one of the most efficient methods, the numerical shooting method [12] gives very accurate results because it integrates the Schrdinger equation directly, though in the numerical sense.
In this case, the wave function of the harmonics oscillator Cosine asymmetric potential is different from that in case of a typical harmonics oscillator potential $\left(\mathscr{V}(x)=(1 / 2) \mu \omega^{2} x^{2}\right)$ (see Figures 2-4). In this case, the time-independent correlation function $(C(S))$ of the harmonics oscillator Cosine asymmetric potential via numerical shooting method and the intensity correlation experiment by reference [23] have the same appearance (see Figures 5-7). From Figures 2(a)2 (c), if the values of the amplitude barrier potential $a$ 


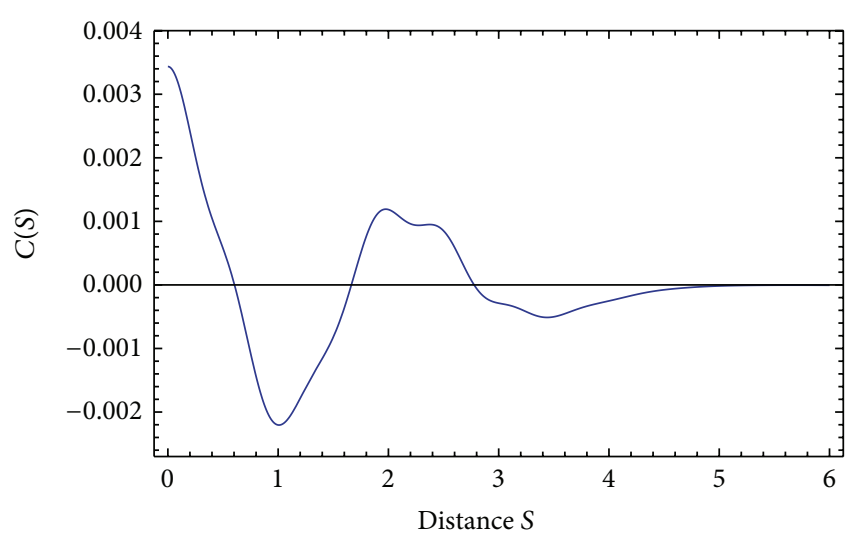

(a) $C(S)=0.0034, C(S)=-0.0022, c=0.3, a=5, b=10$, and $n=1$

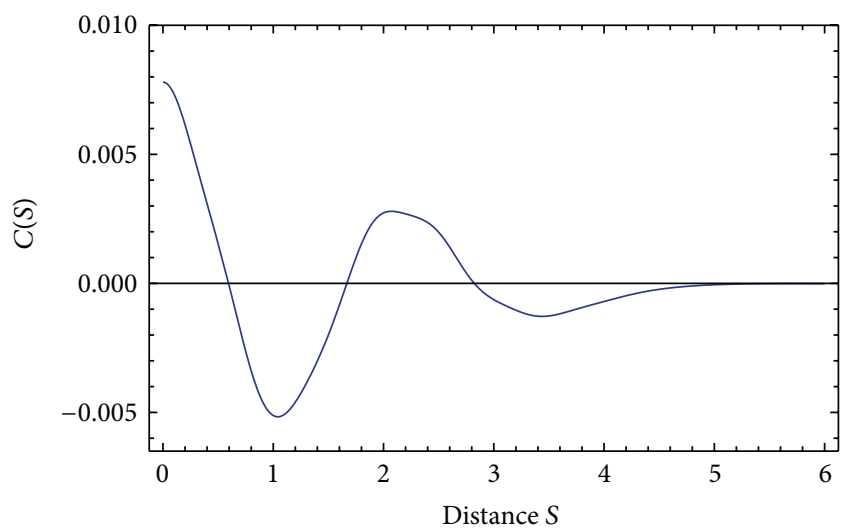

(c) $C(S)=0.008, C(S)=-0.005, c=0.5, a=5, b=10$, and $n=1$

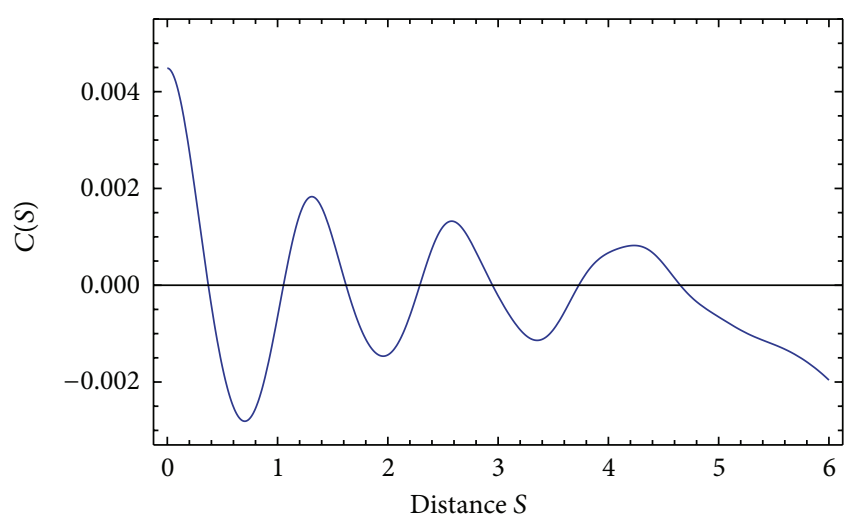

(e) $C(S)=0.0045, C(S)=-0.003, c=0.4, a=5, b=10$, and $n=3$

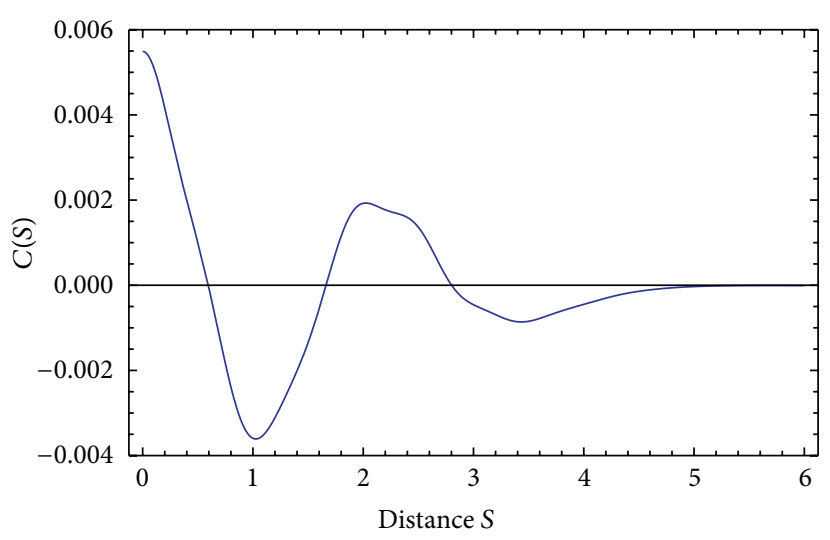

(b) $C(S)=0.0055, C(S)=-0.004, c=0.4, a=5, b=10$, and $n=1$

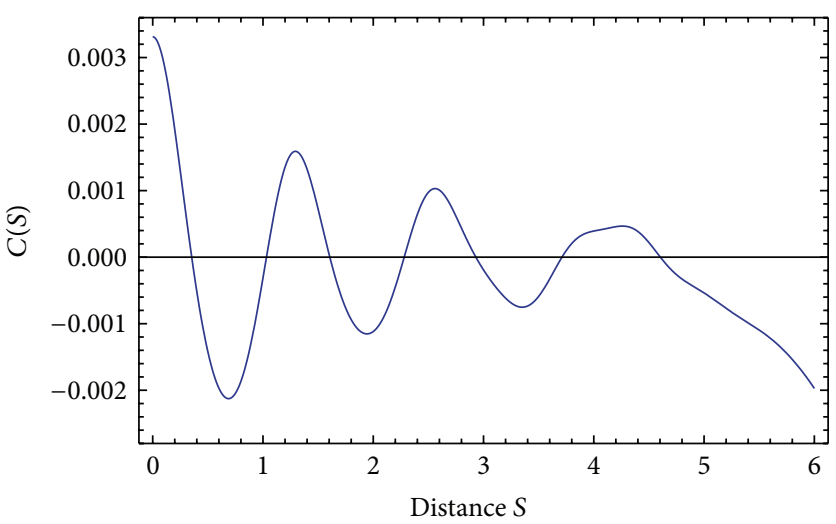

(d) $C(S)=0.0032, C(S)=-0.0022, c=0.3, a=5, b=10$, and $n=3$

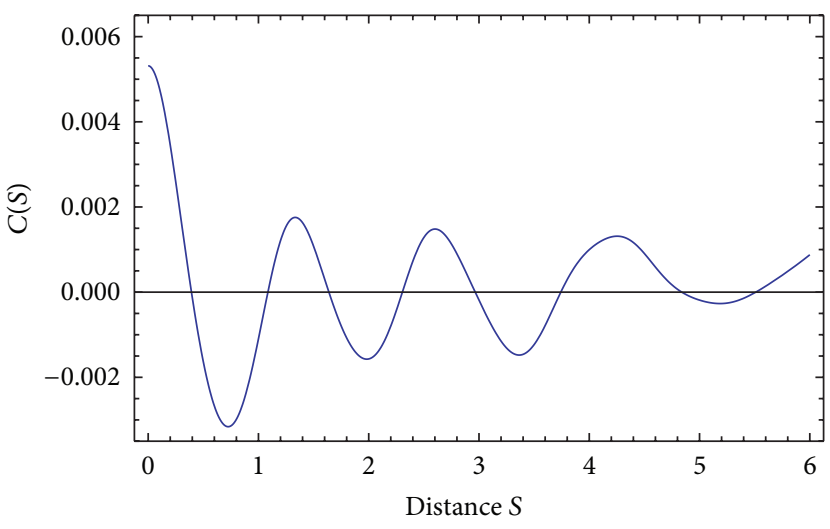

(f) $C(S)=0.0055, C(S)=-0.003, c=0.5, a=5, b=10$, and $n=3$

FiguRE 7: Plot of the time-independent correlation function for the first excited-state energy $(n=1)$ and the third excited-state energy $(n=3)$ in case of the harmonics oscillator Cosine asymmetric potential with varying $c=0.3,0.4,0.5$.

incline, the ground-state energy eigenvalues (En) lessen, but the amplitude of the wave function has supplement and in Figures 5(a)-5(c) the values of the time-independent correlation function $(C(S)$ ) (part of positive) incline and the time-independent correlation function is underdamped motion. From Figures 2(d)-2(f) if the values of $b$ increase, the ground-state energy eigenvalues (En) have supplement, but in Figures 5(d)-5(f) the values of the time-independent correlation function lessen.
From Figures 3(a)-3(f), if the values of the $c$ parameter increase, the ground-state $(n=0)$ and the second excitedstate $(n=2)$ energy eigenvalues (En) lessen, but in Figures $6(\mathrm{a})-6(\mathrm{f})$ the values of the time-independent correlation function have supplement. From Figures 4(a)-4(f), if the values of the $c$ parameter increase, the first excited-state $(n=1)$ and the third excited-state $(n=3)$ energy eigenvalues (En) lessen, but in Figures 7(a)-7(f) the values of the timeindependent correlation function have supplement. From 


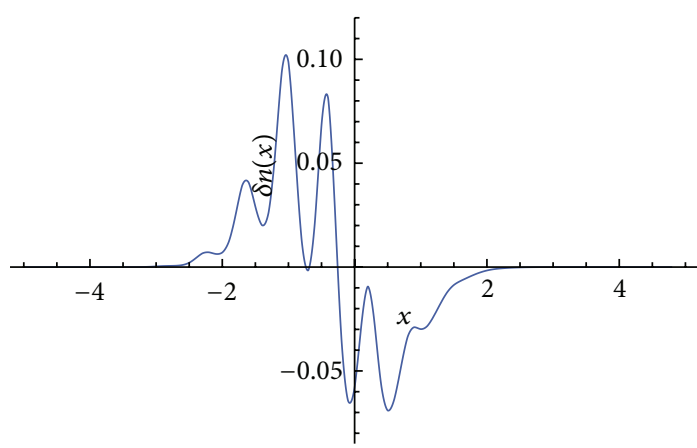

(a) $\delta n(x)=0.10, \delta n(x)=-0.07, a=8, b=10, c=0.4$, and $n=0$

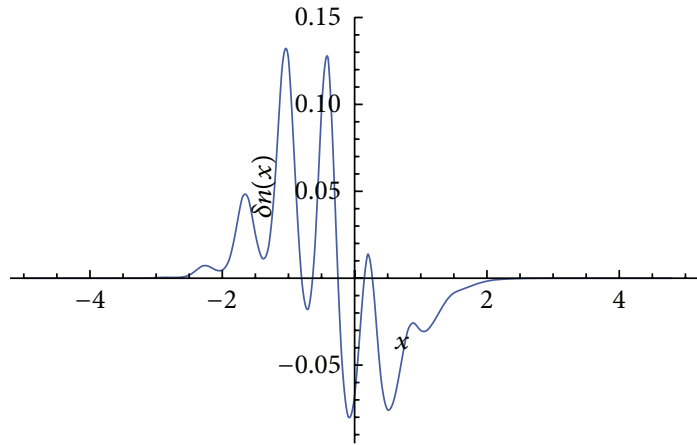

(c) $\delta n(x)=0.13, \delta n(x)=-0.08, a=12, b=10, c=0.4$, and $n=0$

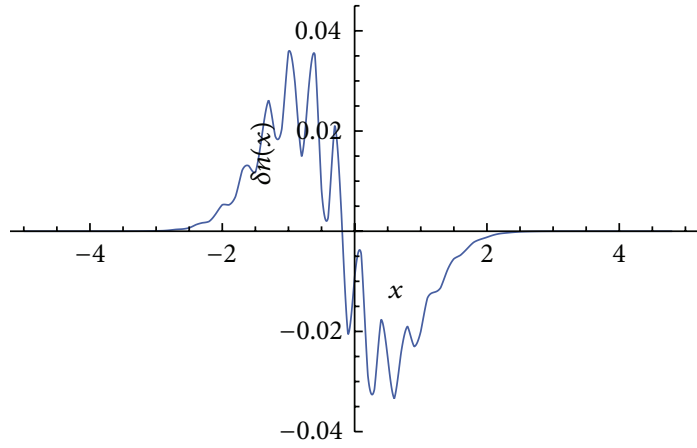

(e) $\delta n(x)=0.035, \delta n(x)=-0.035, a=6, b=18, c=0.2$, and $n=0$

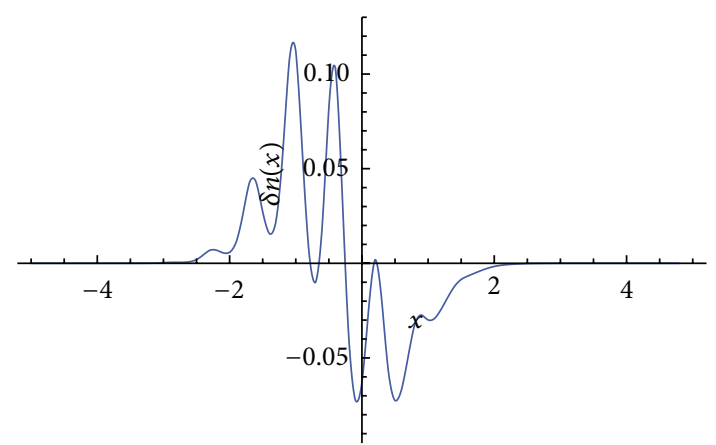

(b) $\delta n(x)=0.12, \delta n(x)=-0.07, a=10, b=10, c=0.4$, and $n=0$

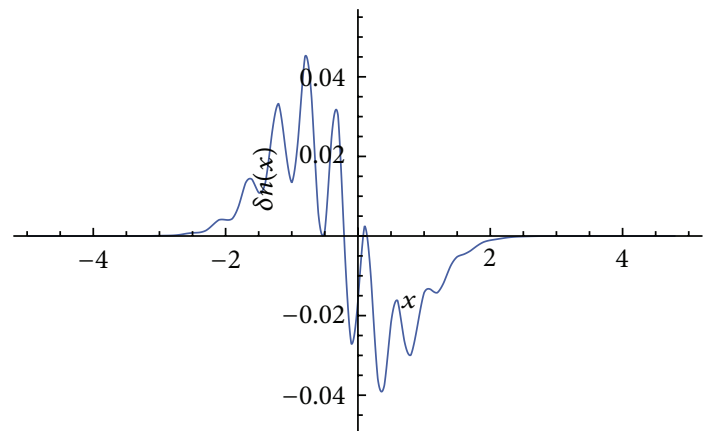

(d) $\delta n(x)=0.045, \delta n(x)=-0.04, a=6, b=14, c=0.2$, and $n=0$

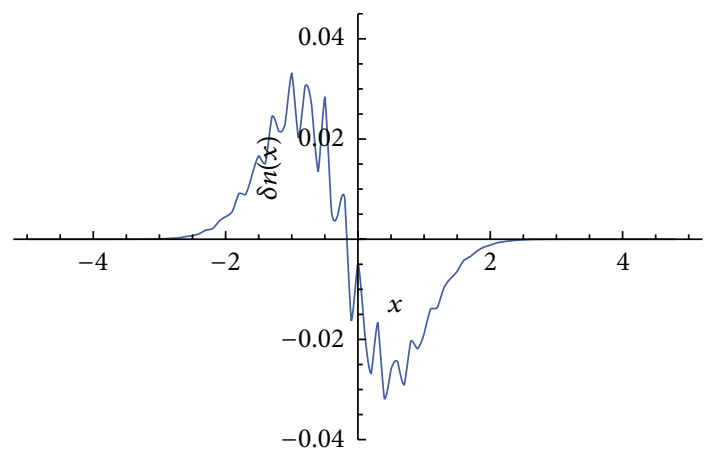

(f) $\delta n(x)=0.033, \delta n(x)=-0.033, a=6, b=24, c=0.2$, and $n=0$

FIGURE 8: Schematic representation for behavior of the time-independent atomic density fluctuation $\delta n(x)=\widetilde{n}(x)-\widetilde{m}(x)$ for the ground-state energy $(n=0)$ in case of the harmonics oscillator Cosine asymmetric potential with varying $c=0.2,0.4$ and $a=6,8,10$.

Figures $8(\mathrm{a})-8(\mathrm{c})$ if the values of the $c, a$, and $b$ parameters increase, the time-independent atomic density fluctuation $(\delta n(x))$ for the ground-state $(n=0)$ has supplement.

\section{Conflict of Interests}

The authors declare that there is no conflict of interests regarding the publication of this paper.

\section{Acknowledgments}

Artit Hutem and Piyarut Moonsri would like to thank the Institute Research and Development Phetchabun Rajabhat
University and Physics Division, Faculty of Science and Technology, Phetchabun Rajabhat University, for partial support.

\section{References}

[1] G. A. Dobrovolsky and R. S. Tutik, "Regularization of the WKB integrals," Journal of Physics A: Mathematical and General, vol. 33, no. 37, pp. 6593-6599, 2000.

[2] M. I. Jaghob, "Perturbation theory for isotropic velocitydependent potentials: bound-states case," The European Physical Journal A-Hadrons and Nuclei, vol. 27, no. 1, pp. 99-103, 2006.

[3] E. Z. Liverts and V. B. Mandelzweig, "Accurate analytic presentation of solution for the spiked harmonic oscillator problem," Annals of Physics, vol. 322, no. 9, pp. 2211-2232, 2007. 
[4] N. Saad, R. L. Hall, and H. Ciftci, "Study of a class of nonpolynomial oscillator potentials," Journal of Physics A: Mathematical and General, vol. 39, no. 24, pp. 7745-7756, 2006.

[5] M. C. Zhang, "Exact bound state solutions of the Klein-Gordon particle in Hulthén potential," Chinese Physics B, vol. 17, no. 9, pp. 3214-3216., 2008.

[6] M. C. Zhang and Z. B. Wang, "Exact solutions of the KleinGordon equation with Makarov potential and a recurrence relation," Chinese Physics, vol. 16, no. 7, article 1863, 2007.

[7] C. R. Handy, H. Hayes, D. V. Stephens, J. Joshua, and S. Summerour, "Application of the eigenvalue moment method to important one-dimensional quantum systems," Journal of Physics. A. Mathematical and General, vol. 26, no. 11, pp. 26352649, 1993.

[8] Z. Cao, "Pressure-induced phase in tetragonal two-dimensional polymeric $C_{60}$," Physical Review A, vol. 63, no. 5, Article ID 054103, 4 pages, 2001.

[9] A. Hutem and C. Sricheewin, "Ground-state energy eigenvalue calculation of the quantum mechanical well $V(x)=1 / 2 k x^{2}+$ $\lambda x^{2}$ via analytical transfer matrix method," European Journal of Physics, vol. 29, no. 3, pp. 577-588, 2008.

[10] H. Ying, Z. Fan-Ming, Y. Yan-Fang, and L. Chun-Fang, "Energy eigenvalues from an analytical transfer matrix method," Chinese Physics B, vol. 19, no. 4, Article ID 040306, 2010.

[11] S. Boonchui and A. Hutem, "Excited-state energy eigenvalue and wave-function evaluation of the Gaussian asymmetric double-well potential problem via numerical shooting method 2," Journal of Mathematical Chemistry, vol. 50, no. 8, pp. 21032119, 2012.

[12] S. Boonchui and A. Hutem, "Excited-state energy eigenvalue and wave-function evaluation of the Gaussian symmetric double-well potential problem via numerical shooting method 1," Journal of Mathematical Chemistry, vol. 50, no. 6, pp. 15821597, 2012.

[13] R. Grobe, K. Rzazewski, and J. H. Eberly, "Measure of electronelectron correlation in atomic physics," Journal of Physics B: Atomic, Molecular and Optical Physics, vol. 27, no. 16, pp. L503L508, 1994.

[14] J. E. Lye, L. Fallani, M. Modugno, D. S. Wiersma, C. Fort, and M. Inguscio, "Bose-Einstein condensate in a random potential," Physical Review Letters, vol. 95, no. 7, Article ID 070401, 4 pages, 2005.

[15] P. Henseler and B. Shapiro, "Density correlations in cold atomic gases: atomic speckles in the presence of disorder," Physical Review A, vol. 77, Article ID 033624, 2008.

[16] N. Cherroret and S. Skipetrov, "Long-range correlations of density in a Bose-Einstein condensate expanding in a random potential," Physical Review Letters, vol. 101, no. 19, Article ID 190406, 2008.

[17] N. Cherroret and S. E. Skipetrov, "Effect of interactions on the diffusive expansion of a Bose-Einstein condensate in a threedimensional random potential," Physical Review A, vol. 79, Article ID 063604, 2009.

[18] L. Beilin, E. Gurevich, and B. Shapiro, "Diffusion of cold-atomic gases in the presence of an optical speckle potential," Physical Review A, vol. 81, Article ID 033612, 2010.

[19] L. Pezze, M. Robert-de-Saint-Vincent, T. Bourdel et al., "Regimes of classical transport of cold gases in a two-dimensional anisotropic disorder," New Journal of Physics, vol. 13, Article ID 095015, 2011.
[20] A. Asaithambi, "Numerical solution of the one-dimensional time-independent Schrödinger's equation by recursive evaluation of derivatives," Applied Mathematics and Computation, vol. 215, no. 12, pp. 4400-4405, 2010.

[21] V. Ledoux and M. van Daele, "Automatic computation of quantum-mechanical bound states and wavefunctions," Computer Physics Communications, vol. 184, no. 4, pp. 1287-1296, 2013.

[22] H. Ciftci, R. L. Hall, and N. Saad, "Exact and approximate solutions of Schrödinger's equation for a class of trigonometric potentials," Central European Journal of Physics, vol. 11, no. 1, pp. 37-48, 2013.

[23] J. Kasprzak, M. Richard, A. Baas et al., "Second-order time correlations within a polariton bose-einstein condensate in a CdTe microcavity," Physical Review Letters, vol. 100, no. 6, Article ID 067402, 4 pages, 2008. 

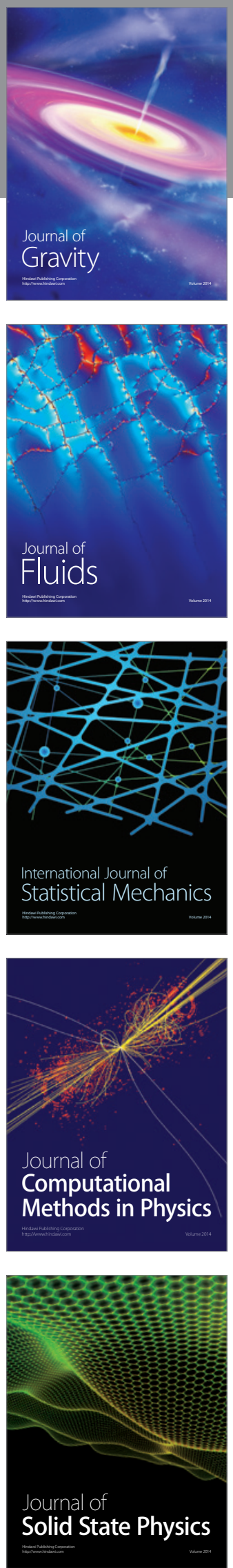

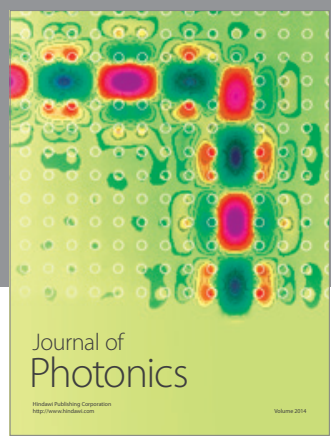

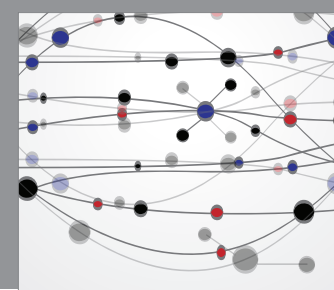

The Scientific World Journal

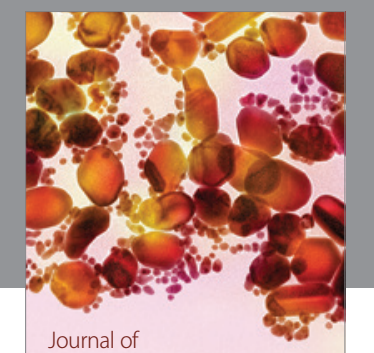

Soft Matter
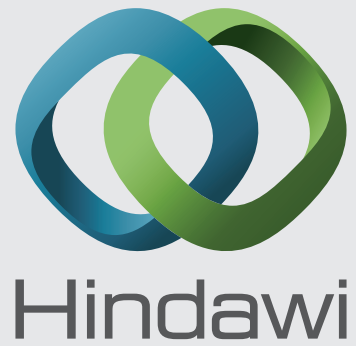

Submit your manuscripts at

http://www.hindawi.com
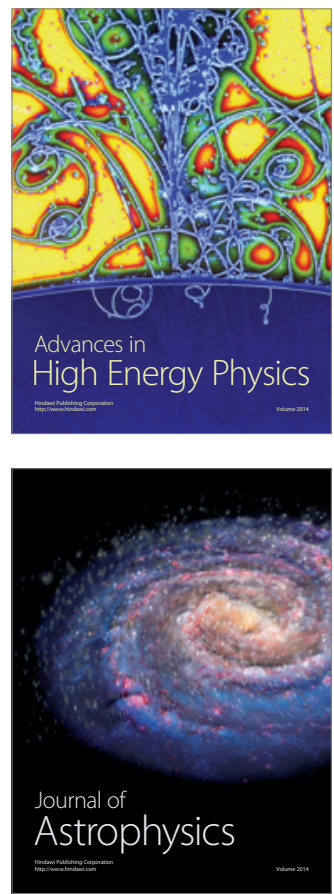
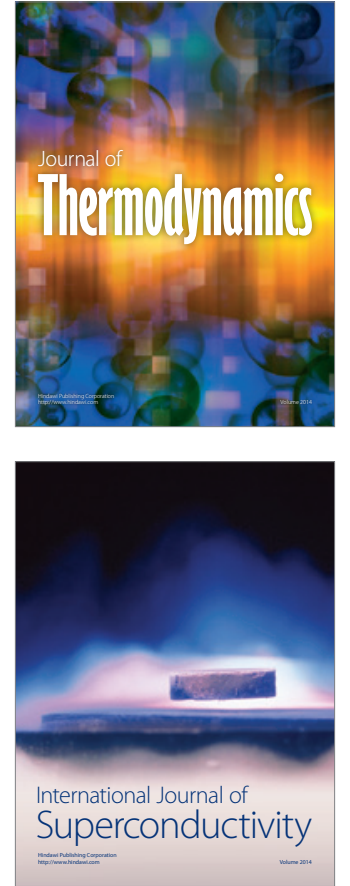
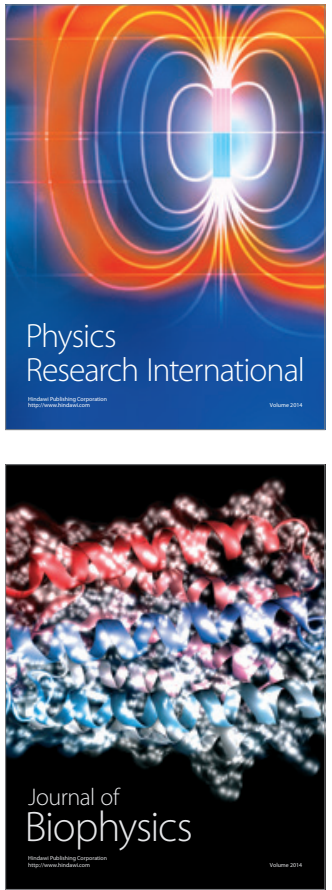
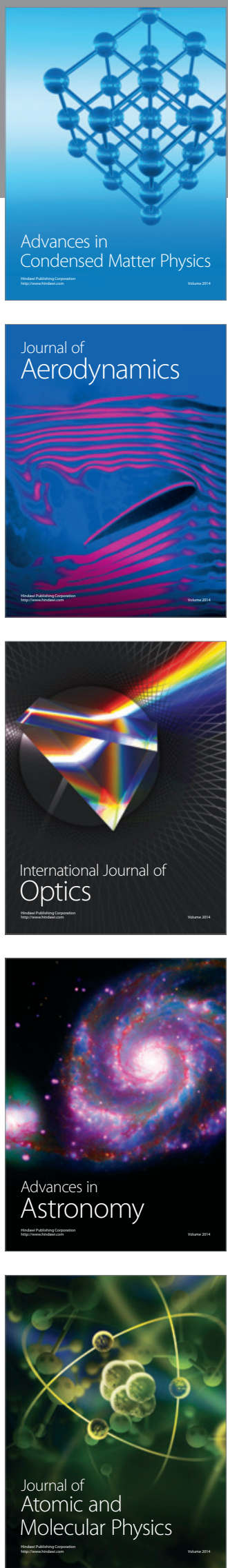